\title{
Characteristics of the membrane of the stereocilia and cell apex in cochlear hair cells
}

\author{
ANDREW FORGE, STEPHEN DAVIES and GARY ZAJIC* \\ EM Unit and Department of Audiology, Institute of Laryngology and Otology, 330-332, Gray's Inn Road, London WC1X 8EE, UK
}

Received 15 October 1987; revised 1 February 1988; accepted 8 February 1988

\begin{abstract}
Summary
Freeze-fracture has been used to examine the membrane of the cell apex and of the stereocilia in cochlear hair cells. The apical (non-stereociliary) membrane of inner hair cells (IHCs) exhibited a lower density of intramembrane particles (IMP) than that of the outer hair cells (OHCs) but in both cell types the apical membrane responded to the effects of filipin. The distribution of IMP and of filipin-induced membrane deformations was uniform over the apical membranes in both IHC and $\mathrm{OHC}$, thus, providing no evidence for local membrane differentiation on the non-stereociliary part of the hair cell apex. The stereociliary membranes of IHC and of OHC differed not only in the density of IMP, but also in their responses to filipin and to tomatin. IHC stereocilia responded intensely to both agents. OHC stereocilia showed a significantly lower density of filipin-induced lesions and appeared almost unaffected by tomatin. This suggests that the OHC stereocilial membrane may be structurally specialized. The membrane at the apical end of stereocilia appeared to be differentiated from the membrane of the stereociliary shaft. The tip region was free of the usual IMP and showed no filipin-induced lesions. The differentiation at the apical end was also apparent in samples which have been rapidly frozen without prior chemical fixation or cryoprotection, showing that the particle-free area was not an artefact induced by glutaraldehyde fixation. Close examination of the membrane at the apical-most tip of the stereocilium revealed the presence of a small number of large particles of $10.5-11.0 \mathrm{~nm}$ diameter. The occurrence of membrane differentiation localized to the tip of the stereocilium may be consistent with the suggestion that transduction channels in hair cells are situated at this point.
\end{abstract}

\section{Introduction}

Hair cells are the stimulus receptors of vertebrate balance and hearing organs and participate in the transduction of mechanical stimuli to neural output. Their name derives from the fact that at the apex of each cell is a bundle of rigid, erect projections (hairs), the stereocilia. The term 'cilia' is a misnomer in this case since stereocilia are more akin to microvilli, being formed of regularly packed actin fabrils (Flock \& Cheung, 1977; DeRosier et al., 1980) enclosed within extensions of the apical plasma membrane. In all hair cells, the bundle of stereocilia is highly organized (Tilney \& Tilney, 1984) with a progressive increase in the length of the stereocilia in one particular direction across the bundle. Mechanical displacement of the stereocilia along this line of orientation, i.e. towards and away from the longest stereocilia, induces changes in intracellular potentials (Hudspeth, 1983).

In the mammalian cochlea, the hair cells are contained within the organ of Corti, which is coiled into a spiral, and two types of hair cells are recognized: the outer and the inner hair cells (OHCs,

IHCs). In most mammals there is a single row of IHCs and three rows of OHCs. The hair bundle is formed of 3-5 rows of stereocilia, the length of the stereocilia increasing in each successive row from inside to out. In IHCs the stereocilial rows are in an approximately straight line oriented along the long axis of the organ of Corti, whilst on OHCs, the stereocilia form a ' $W$ ' (or ' $\mathrm{M}^{\prime}$ ) shape. There are other significant morphological differences between IHCs and OHCs (see for example, Lim, 1986) and their innervation patterns are also very different. Approximately $95 \%$ of all afferent innervation arises from IHCs and each IHC synapses with several different nerve fibres (Spoendlin, 1974). On the other hand, OHC innervation is predominantly efferent. Recently, it has also been shown that isolated OHCs are capable of undergoing contraction (Brownell, 1984; Zenner et al., 1985). These and other physiological (Russell et al., 1986) and biophysical (Kemp, 1982) observations have led to suggestions that the IHC is the sensory cell in the cochlea while the OHCs, having detected a stimulus, actively affect cochlear mechanics to provide an

*Permanent address: Kresge Hearing Research Institute, University of Michigan, 1301 East Ann Street, Ann Arbor, Michigan 48109, USA.

0300-4864/88 \$03.00+12 (C)1988 Chapman and Hall Ltd. 
amplification of the signal reaching the IHC, thereby increasing sensitivity.

Until recently, the precise relationship between stereocilial movement and activity within the hair cell was not clear, but evidence is now accumulating that the 'transduction channels' through which changes in intracellular potentials are affected, are located on the stereocilia, possibly at their tips. The estimated number of transduction channels on each cell coincides approximately with the number of stereocilia on the cell (Russell, 1983; Hudspeth, 1986). In frog vestibular hair cells, which are thought to function in a similar manner to cochlear hair cells, determination of current flow at different locations along the length of the stereocilial bundle (Hudspeth, 1982) has shown maximal flow at the tip, suggesting this to be the site of the transduction channel. At the morphological level, high resolution scanning and transmission electron microscopy (Pickles et al., 1984; Furness \& Hackney, 1985) have shown that at the tip of stereocilia there is a fine fibril which links a short stereocilium to a longer one behind. It has been proposed (Pickles et al., 1984) that this link acting mechanically gates the transduction channel.

In the present work freeze-fracture has been used to examine the membranes at the apex of cochlear hair cells to discover whether these membranes possess characteristics of relevance to understanding hair cells functioning. Of particular interest was whether the presumed functional difference between IHCs and OHCs is reflected in differences in the membrane characteristics at the cell apex and whether it was possible to identify any local membrane differentiations perhaps related to the sites of transduction channels. Previous freezefracture studies of cochlear hair cells have shown that the apical plasma membrane is morphologically distinguishable from the lateral and basal membrane of the hair cells (Gulley \& Reese, 1977; Forge, 1986) and indicated some differences in the membranes structure of equivalent membrane regions between IHCs and OHCs. A further consideration in the present work was that high resolution electron microscopy studies of stereocilia have drawn attention to the possible vulnerability of details of stereocilial morphology to procedures used in processing cochlear tissue for electron microscopy (Pickles et al., 1984). To investigate this in part, non-chemically fixed, rapidly-frozen preparations of the intact organ of Corti and of isolated OHCs have been examined; preliminary results of these studies are also presented here.

\section{Materials and methods}

Young adult, pigmented and albino guinea pigs and young adult gerbils were used. All animals were free of ear infection. Animals were killed by anaesthetic overdose, decapitated, the acoustic bullae removed and the cochleae widely exposed.

\section{Standard preparation for freeze-fracture}

Most of cochleae used in this study were prefixed before freezing before freeze-fracture. Tissue was fixed in $2.5 \%$ glutaraldehyde in $0.1 \mathrm{M}$ cacodylate buffer with $3 \mathrm{M} \mathrm{CaC1}_{2}$, $\mathrm{pH} 7.3$, total osmolarity $550 \mathrm{~m} 0 \mathrm{sm}$. The fixative was equilibrated to room temperature before use and was gently injected into the cochlea through openings at the cochlear apex and at the base. The whole bulla was then immersed in fixative and fixation continued for a total of $1.5 \mathrm{~h}$ at room temperature with constant slow rotation.

Following fixation, short segments of the organ of Corti were dissected out under $0.1 \mathrm{M}$ cacodylate buffer. The tissue was then infiltrated with $25 \%(\mathrm{v} / \mathrm{v})$ glycerol and mounted into the holes in gold double-replica specimen supports using a yeast in glycerol paste as mountant to enable orientation of the tissue. Samples were frozen by plunging into solidifying Freon 22 cooled in liquid nitrogen.

\section{Thin sections}

Segments of the organ of Corti were post-fixed in $1 \% \mathrm{OsO}_{4}$ in $0.1 \mathrm{M}$ cacodylate buffer for $1.5 \mathrm{~h}$ at room temperature, rinsed in distilled water and then en bloc stained in $1 \%$ aqueous uranyl acetate for $3 \mathrm{~h}$ in the dark at room temperature before dehydration and embedding in araldite.

\section{Filipin and tomatin treatment}

In some preparations, glutaraldehyde-fixed tissue was treated with filipin (a gift from Dr J. E. Grady, Upjohn Ltd and from Sigma) or with tomatin (Sigma) to enable localization of membrane sterols (Severs \& Robenek, 1983). Both agents were dissolved in dimethyl sulphoxide (DMSO); filipin was used at a final concentration of $100 \mathrm{mg} \mathrm{ml}^{-1}, 1 \%$ DMSO and tomatin at $150 \mathrm{\mu g} \mathrm{ml}^{-1}, 1 \%$ DMSO in $0.1 \mathrm{M}$ cacodylate buffer (Forge, 1985). Incubation was continued for $18 \mathrm{~h}$ at room temperature before the tissue was prepared for freeze-fracture and thin sectioning as above. Control tissue for these preparations was incubated similarly in $1 \%$ DMSO in $0.1 \mathrm{M}$ cacodylate buffer and ultimately showed no obvious difference from normal non-treated tissue.

\section{Rapid-freezing of intact organ of Corti}

The bullae were removed from the animal and immediately transferred to Hank's balanced salt solution (HBSS), $\mathrm{pH}$ 7.3 , osmolarity adjusted to $300 \mathrm{~m} 0 \mathrm{sm}$. The unfixed organ of Corti was then dissected out and individual pieces mounted on single thin copper specimen supports or between two such supports to form a sandwich. The copper supports had been exposed briefly to concentrated nitric acid before use to roughen the surface and enhance adhesion of the tissue. Excess fluid was carefully blotted away then the tissue on its support was plunge-frozen in a constantly stirred mixture of 3:1 propane:isopentane cooled by liquid nitrogen. Rapid plunge-freezing was achieved using a device designed for slam-freezing by Boyne (Philips 
\& Boyne, 1984), slightly adapted so that samples could be plunged into cryogens.

\section{Isolated outer hair cells}

OHCs were isolated by the method described by Zajic \& Schacht (1987). Briefly, the bullae were removed to HBSS and the apical two turns of the cochlea dissected out. These were incubated for $10 \mathrm{~min}$ at room temperature in $1 \mathrm{mg} \mathrm{ml}^{-1}$ collagenase in HBSS then placed in a drop of fresh HBSS. The hair cells were manually dissociated from the basilar membrane with fine tungsten wire probes and collected into a small drop of fresh medium. Droplets of $1 \mu \mathrm{l}$ of this suspension of isolated cells were placed on surface-roughened copper specimen supports and some were sandwiched between two such copper plates. Samples were rapidly plunge frozen as above.

\section{Freeze-fracture}

Freeze-fracture replicas were prepared in a Balzers BAF 400 apparatus, fitted with a liquid nitrogen-cooled anticontamination shroud, using a double replica device to fracture samples or the knife to cleave the specimen as appropriate. Fracturing or cleaving was performed at $158 \mathrm{~K}$ at a pressure of $<10^{-6} \mathrm{mBar}$. Most samples were undirectionally shadowed with platinum-carbon from an angle of $45^{\circ}$, but some were rotary shadowed from $25^{\circ}$ with the specimen rotating at $\sim 60$ r.p.m. during both shadowing and carbon backing.

\section{Results}

The apical membranes of the hair cells were readily identified in freeze-fracture replicas by the images of the cross-fractured insertions of the stereocilia (Figs. 1 and 2) which formed the characteristic patterns of the stereocilial bundles: on IHCs (Fig. 1) the stereocilia cross-fractures were in an approximately straight line whilst on OHCs (Fig. 2), they were in a W-shaped configuration. The clarity of the images of these patterns provided an opportunity to examine some details of the organization of the stereocilial bundle. On OHCs, the stereocilia were regularly arranged in three parallel rows along the two arms of the ' $W$ '. The spacing between each successive row appeared to be uniform, as did the separation between stereocilia in a single row. On IHCs the bundle was less regular. The number of rows was difficult to enumerate exactly since the innermost (i.e. shortest and thinnest) stereocilia did not appear to be in an obviously regular arrangement. However, on most IHCs at least four complete rows could be identified, the outermost two of which formed an almost straight line, whilst the more inward appeared as two joined curves. The separation of IHC stereocilia also was not as regular as that on OHCs. The outermost two rows (i.e. the longest stereocilia) were relatively widely separated from each other and from the more inner stereocilia.
Examination of the details on the fracture faces of the hair cell apical membranes showed some differences between IHCs and OHCs. On IHCs, the p-faces of the apical membrane (Fig. 3) possessed relatively few intramembrane particles (IMP) at a density of $\sim 120 \mu \mathrm{m}^{-2}$ and there appeared to be at least two types of particles as judged by size. The e-faces of the IHC apical membrane (Fig. 4) were almost completely devoid of IMP. On the apical membrane p-faces of OHCs (Fig. 5) there also appeared to be particles of two different sizes but IMP were much more numerous than on IHCs, present at a density of $\sim 350 \mu^{-2}$. Some particles were also present on the e-faces of the OHC apical membrane (Fig. 6). The majority of these e-face particles appeared to be similar to the larger particles on the p-face. On both IHCs and OHCs, the distribution of IMP was uniform over the entire apical membrane fracture face and there was no indication of any local membrane differentiation. Following exposure of the tissue to filipin, the apical membranes of both IHC and $\mathrm{OHC}$ showed characteristic discrete membrane deformations (filipin-induced 'lesions') over the fracture faces. As exemplified in Fig. 7, lesions were uniformly distributed over the entire fracture face.

The fracture faces of the membranes of the stereocilia of both IHC (Fig. 8) and OHC (Fig. 9) in pre-fixed samples had an even contour. In a fashion similar to that of the apical membranes, IMP on the membranes IHC stereocilia were less numerous than on those of the OHC. IHC and OHC stereocilial membranes also consistently showed differing responses to both filipin and tomatin treatment. In thin sections of filipin-treated tissue, the stereocilial membranes of IHCs were almost entirely deformed by numerous filipin-induced corrugations (Fig. 10) but on OHC stereocilia, such corrugations were dispersed (Fig. 11). These observations were confirmed in freeze-fracture replicas. IHC stereocilial membranes showed filipin lesions closely packed over the fracture faces (Fig. 12) at a density of $\sim 235 \mathrm{um}^{-2}$; on OHC stereocilia, the density of lesions was much lower (Fig. 13): $\sim 140 \mu \mathrm{m}^{-2}$. Statistical analysis of the mean density of filipin lesions from 15 OHC stereocilial bundles in three different preparations compared with that from 11 IHC stereocilial bundles in the three preparations, showed this difference in the lesion density between IHC and OHC stereocilia to be significant (at $p=0.05$ ). With tomatin, the pattern of response of $\mathrm{IHC}$ and $\mathrm{OHC}$ stereocilial membranes was similar to that with filipin. Tomatin appeared to cause quite severe disruption of the membranes of the stereocilia on IHCs; the membranes did not carry the fracture plane readily so that, frequently, cross-fractures of the stereocilia rather than membrane fracture faces were exposed (Fig. 14). However, tomatin appeared to 
have little effect on the membranes of $\mathrm{OHC}$ stereocilia (Fig. 15).

In normal freeze-fracture preparations, in both IHC and $\mathrm{OHC}$, the membrane at the apical tip of the stereocilium consistently appeared almost smooth and free of the normal IMP (arrows Figs. 8 and 9). Sometimes a similar 'bare' area could be identified on one side of the stereocilium. In filipin-treated samples, the apical ends of stereocilia were lesionfree (Figs. 12, 16). Thin sections showed this lesion-free area to correspond with the region of sub-membrane thickening and point of insertion of the extracellular cross-link at the tip of the stereocilium (Fig. 10). Close examination of the tipmost region in freeze-fracture replicas showed a small number of large particles on the $\mathrm{p}$-fracture faces (Figs. $9,16)$ which, from complementary replicas, could be seen to correspond with pits in the e-fracture faces (Fig. 17). Following rotary replication of normal freeze-fractured samples, the large tip particles were clearly resolved and could be easily distinguished from the normal IMP (Fig. 18). They had a diameter of $10.5-11.0 \mathrm{~nm}$.

In nón-chemically fixed, rapidly frozen samples of the intact organ of Corti, the stereocilial membranes showed similar features to those in pre-fixed, cryoprotected samples. The membrane contour was even, showing no convolutions, and at the apical end, the membrane fracture face appeared smooth and free of the normal IMP (Fig. 19). In preparations of isolated $\mathrm{OHCs}$, again frozen without any pretreatment, the true surface of the stereocilium was occasionally revealed. This surface was smooth (i.e. particle-free) but granule-like irregularities were dispersed over it (Fig. 20, 21). This appearance can be compared with the fracture face of the stereocilial membrane in isolated OHCs (Fig. 22). This, like those of both pre-fixed and non-prefixed intact organ or Corti preparations, showed an even membrane topography and exhibited IMP randomly dispersed over the membrane of the stereociliary shaft.

\section{Discussion}

The tissues of the inner ear are relatively inaccessible and are highly differentiated. In consequence, ultrastructural studies of the organ of Corti assume considerable value as a ready means of determining the characteristics of the individual cell types. It is, therefore, of some importance to consider the possible formation of artefacts during the preparation of tissue for electron microscopy, especially as some recent studies have suggested that significant fine details of the structure are vulnerable to the processing procedures used routinely for thin sectioning and scanning electron microscopy (Pickles et al., 1984). Of particular relevance is the contention that the membranes of the stereocilia in 'wellpreserved' specimens is finely convoluted, appearing as surface roughness in SEM preparations, rather than being smooth surfaced as is normally found in routine preparations. In the present work, stereocilia always appeared to have a smooth contour both in glutaraldehyde-fixed specimens and in nonchemically treated, rapidly frozen samples. This indicates that routine processing procedures do not induce significant distortion of the membrane.

Irregular roughness was, however, ôbserved on the extracellular surface of the stereocilium in the preparations of isolated outer hair cells. Thus, the fine roughening observed in high resolution SEM studies may represent some extracellular feature such as the cell coat known to be present at the apices of cochlear hair cells (Santi \& Anderson, 1986). In the chick vestibular hair cells, rapidly-frozen, deepetched preparations have shown the cell coat around the stereocilia to be fibrillar in nature (Hirokawa \& Tilney, 1982), but the surface of the stereocilia in the isolated cochlear OHCs shown here did not show this structure. It is possible therefore, that the surface roughness is artefactual, resulting from either distortion due to inadequate freezing or the deposition of a eutectic from the culture medium in which the cells

Fig. 1. Apical membrane of an inner hair cell from the upper basal coil of the cochlea. The images of the cross-fractured insertions of the stereocilia are clearly visible. The thinnest stereocilia are towards the inside of the cochlear spiral. Fractured; $\times 7820$.

Fig. 2. Apical membrane of an outer hair cell at the same point in the cochlea as the IHC in Fig. 1. The base of the ' $W$ ' is towards the outside. The continuity of the stereocilial and apical membranes is indicated at the arrow. Fractured; $\times 9660$.

Fig. 3. Inner hair cell apical membrane $p$-fracture face. IMP are widely distributed. Within the cross-fractured stereocilia the relatively small number of fibrils comprising the stereociliary shaft at the point of insertion into the cell can be seen. Fractured; $\times 58650$.

Fig. 4. Inner hair cell apical membrane e-fracture face from the complementary replica to that shown in Fig. 3 . The face is almost totally free of particles. Fractured; 58650.

Fig. 5. Outer hair cell apical membrane p-fracture face. The cell is from the same region of the cochlea as the IHCs in Figs. 3 and 4. Fractured; $\times 58650$.

Fig. 6. Outer hair cell apical membrane e-fracture face from the complementary replica to that in Fig. 5. Fractured; $\times 57500$.

Fig. 7. Apical membrane of an $\mathrm{OHC}$ following filipin treatment. Filipin-induced lesions are quite closely packed and distributed over the entire fracture face. Fractured; $\times 10212$. 

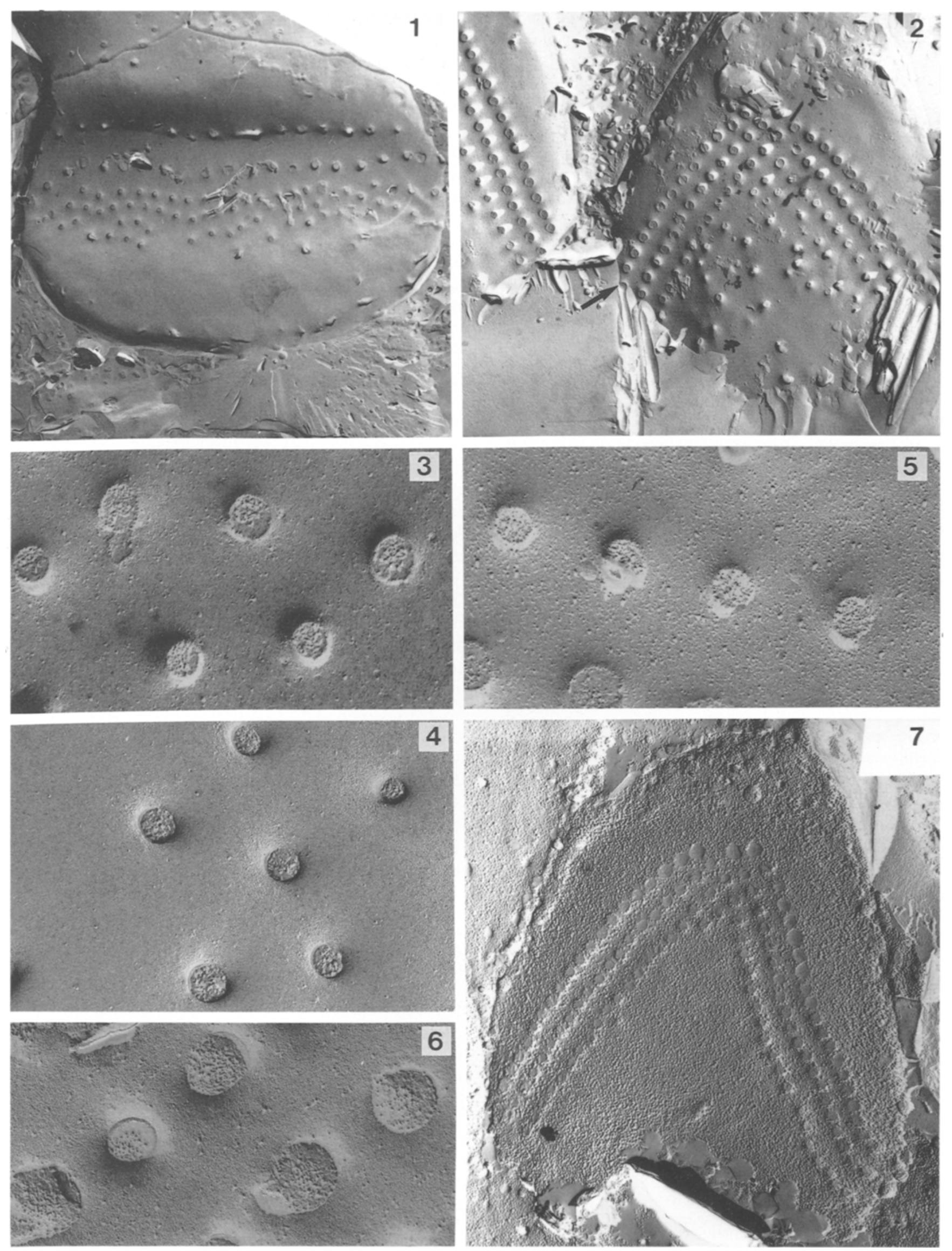
were frozen. However, the fracture faces of the stereocilial membranes in the same isolated hair cell preparations did not show any obvious freezing artefacts (Fig. 22). Further, the fractured surfaces were not intentionally etched for any prolonged period and it has been argued (Hirokawa \& Tilney, 1982) that obvious condensates of salt appear on exposed surfaces only after considerable periods of etching. It seems more likely that the observed irregularities on the extracellular surface of the stereocilia in the isolated cells represent a disruption of the cell coat caused during the isolation procedure. Scanning electron microscopy has also indicated similar abnormalities of the surface features of the stereocilia in isolated OHCs (A. F. and G. Z. unpublished observations).

$\overline{\mathrm{A}}$ further consideration in relation to artefact formation in freeze-fracture studies is that there is evidence that glutaraldehyde fixation may induce re-arrangement of membrane components leading to the production of artefactual details on fracture faces (Hasty \& Hay, 1978; Chandler, 1984). However, for the stereocilial membrane, there was little obvious difference between the glutaraldehyde-fixed preparations and the non-pretreated, rapidly frozen samples in the details present on the fracture faces. Further examination of rapidly frozen tissue is necessary to confirm these observations, but is would seem that pretreatment of the tissues of the organ of Corti before freeze-fracture does not cause identifiable alterations to details of membrane structure. This makes it possible to approach assessment of the results with greater confidence.

Following freeze-fracture of the organ of Corti, four structurally distinct regions of the plasma membrane surrounding a hair cell can be identified (Gulley \& Reese, 1977; Forge, 1986): the apical membrane, which faces potassium-rich endolymph; the tight junction region; the lateral membrane which is in contact with the sodium-rich fluids in the spaces of the organ of Corti; and the membranes of the syn- aptic regions. The present results, in confirmation of earlier observations (Gulley \& Reese, 1977), show that the distinguishing feature of the fracture faces of the apical membranes of both IHCs and OHCs is a relatively low density of IMP. However, as the density of IMP on IHCs is 3-4-fold lower than on OHCs, there may be some difference between the two cell types in the structure of the membrane in this region of the cell. Whether this difference in IMP density is a reflection merely of differing relative quantities of similar components or represents a more fundamental compositional difference is not known, but it would seem to indicate a difference in the functional activity of the apical membranes of IHCs and OHCs.

The present work also extends previous investigations by emphasizing differences between the cochlear hair cell types at the level of the stereocilial membrane. This was seen particularly in the differing responses to filipin and tomatin. Some care is needed in the interpretation of these results (Severs \& Robenek, 1983) since with filipin, a number of factors may affect its interaction with cholesterol or the development of the reaction into visible, discrete deformations of the membrane. Consequently, although the formation of lesions indicates the presence of cholesterol, the density of lesions is not an accurate reflection of sterol concentration. However, consistent differences between membranes in their response to filipin does imply differences in their structure and/or composition. The use of tomatin may overcome some of the problems associated with filipin as tomatin often produces a visible reaction with cholesterol in membrane regions which are resistant to filipin (Severs \& Robenek, 1983). Therefore, the consistently differing response of the IHC and of the OHC stereocilial membranes to both filipin and tomatin may well indicate that the cholesterol content in the stereocilia of OHCs is lower than that of IHCs.

As judged from the responses to probes for membrane cholesterol, the membranes of the $\mathrm{OHC}$

Fig. 8. IHC stereocilia in fixed, cryoprotected specimen. The arrow indicates particle-free region at the apical end. Fractured; $\times 51600$.

Fig. 9. OHC stereocilia in fixed cryoprotected specimen. Arrow indicates tip region free of normal IMPs, but with a few large particles. Fractured; $\times 68800$.

Fig. 10. Thin-section of IHC stereocilium in filipin-treated preparation. The stereocilial membrane shows numerous filipin-induced corrugations covering the entire membrane except for the tipmost region. Arrow indicates the lesion-free tip where a fine cross-linking fibril attaches to the shorter stereocilium and a subplasmallemal electron dense region is present. $\times 61920$.

Fig. 11. Thin section of $\mathrm{OHC}$ stereocilium in filipin-treated preparation; same section as the IHC shown in Fig. 10. Filipin-induced membrane corrugations are more dispersed than on the IHC membrane. The membrane region where lateral cross-links between stereocilia attach to the stereociliary shaft is free of corrugations. $\times 61920$.

Fig. 12. Freeze-fracture of IHC stereocilia following filipin treatment. The membrane is densely covered with filipin-induced membrane deformations, but at the apical end, lesions appear to be absent (arrow). $\times 28980$.

Fig. 13. Freeze-fracture of OHC stereocilia following filipin treatment. This is from the same piece of tissue as the IHC shown in Fig. 10. The density of lesions is less than on the IHC stereacilia. $\times 30960$. 

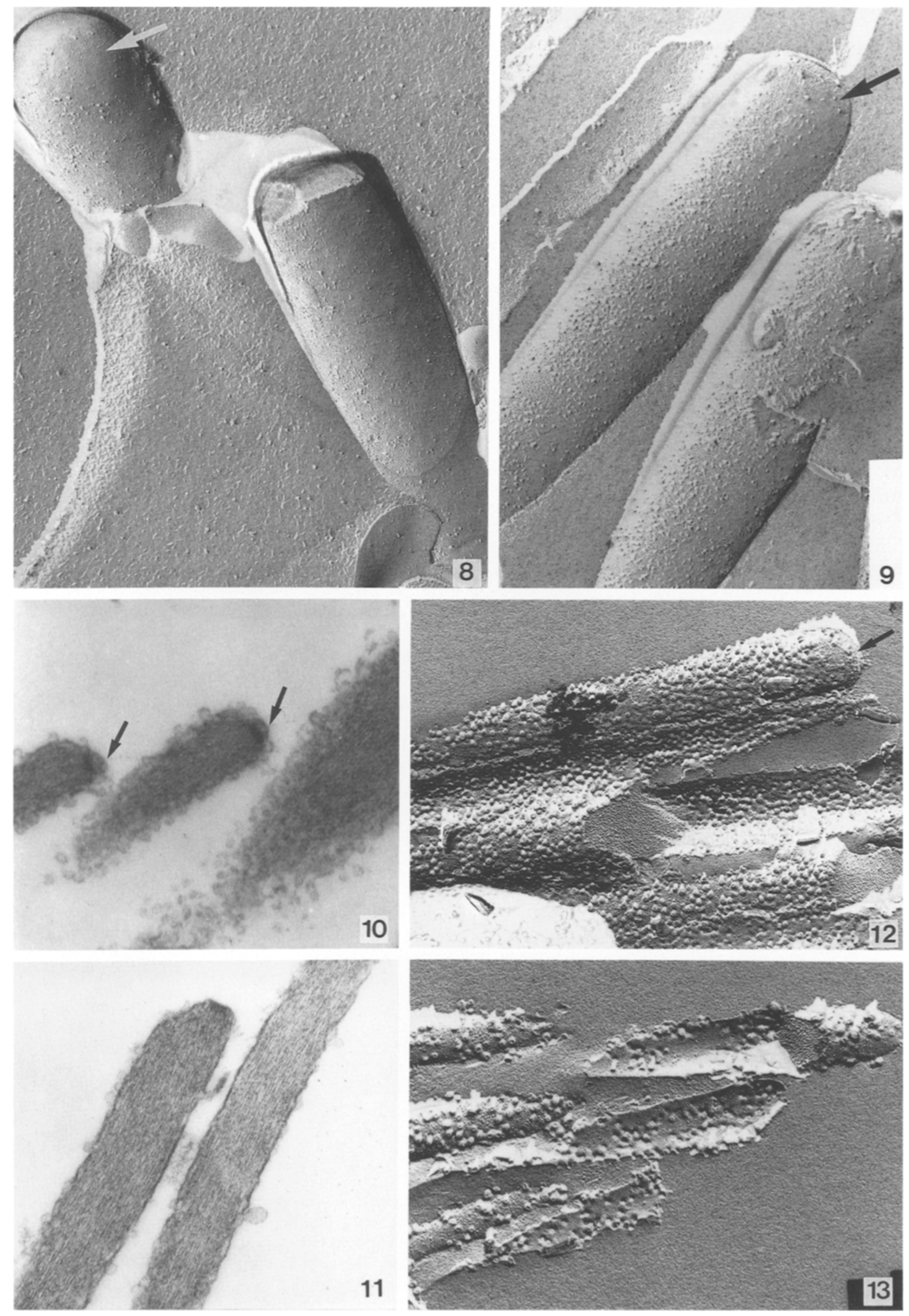

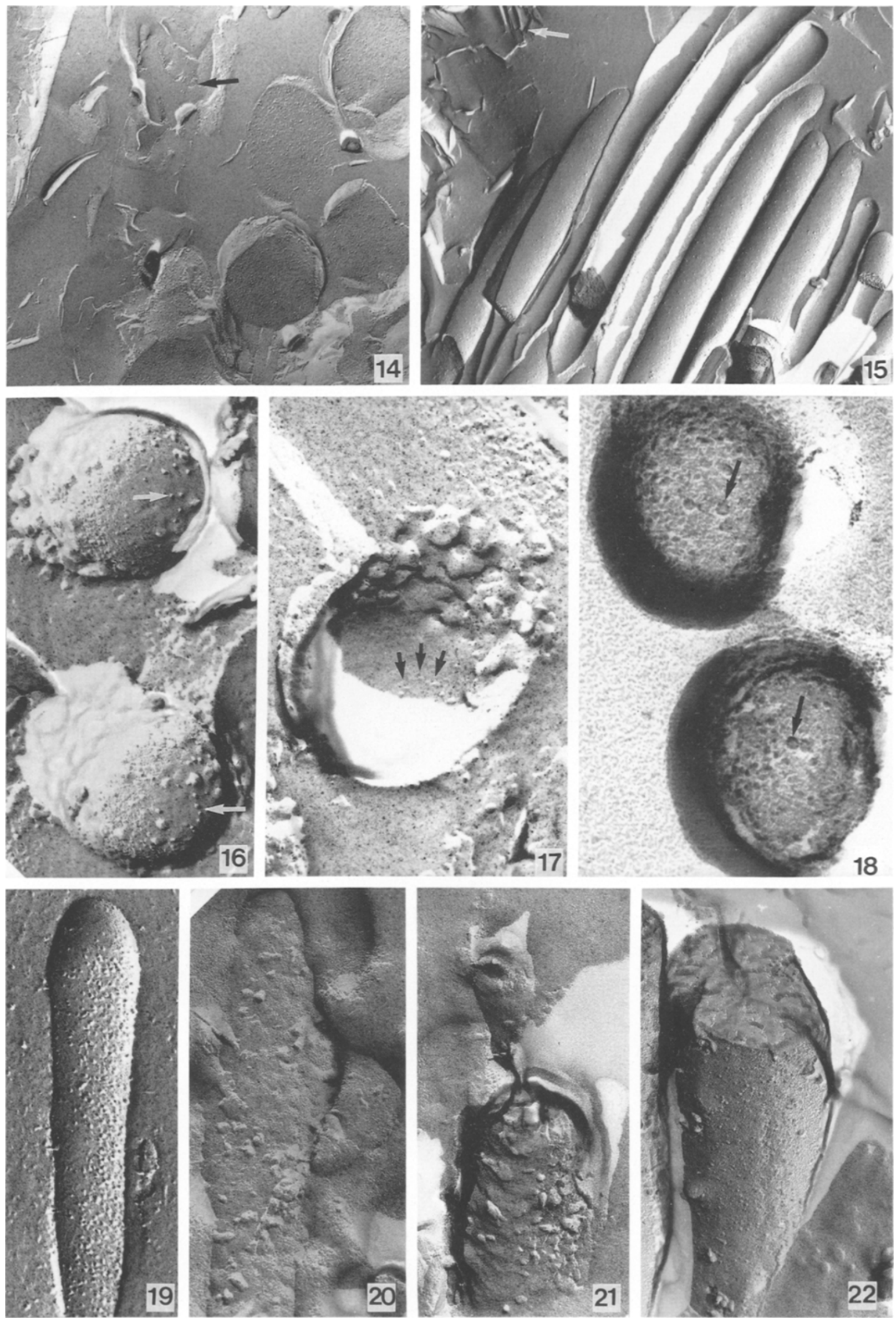
stereocilia differ not only from those IHCs but also from the stereocilia of vestibular hair cells. Stereocilial membranes of vestibular hair cells respond intensely to both filipin and tomatin (A. F. in preparation) and in this respect the cochlear IHCs appear to be more similar to vestibular hair cells than to cochlear OHCs. These observations may indicate a specialization of the stereocilia on OHCs involving the maintenance within the membrane of a cholesterol level lower than that of the stereocilia of other hair cell types and which may be related to the presumed functional specialisation of the OHCs.

The consistent findings that the membrane at the apical end of the stereocilium is free of the normal IMP present on the stereociliary shaft and that this region does not show a visible response to filipin, suggest that there may be a local structural specialization of the membrane at the tip of the stereocilium. Other structural specializations at the tips of at least the shorter stereocilia on a hair cell have been recognized by SEM and thin sectioning. A fine fibril is attached at the tip of these stereocilia which cross-links to a longer stereocilium behind and, at the site of attachment of the fibril to the tip, there is a localized thickening of the membrane (Pickles et al., 1986). The present results show that that region at the tip of the stereocilium which appears resistant to the deforming activity of filipin corresponds to the point of attachment of the tip link and the area of membrane thickening on these shorter stereocilia. However, SEM and thin sections also suggest that the longest stereocilia on a hair may be different from the shorter ones as they do not appear to possess a tip link. In the freeze-fracture replicas, specialization of the membrane at the tip of the stereocilium was a common finding and no differences between stereoci- lia could be detected. It is likely that the majority of stereocilia exposed on fracture were the shorter type as these constitute the majority of the stereocilial bundle ( $\sim 66 \%$ on OHCs; $\sim 75 \%$ on IHCs), but, because unequivocal identification of stereocilial type (i.e. short or long) in freeze-fracture replicas is difficult, any assessment of possible differences between the different stereocilia on a single hair cell could not be made.

The fact that the membrane at the apical tip of the stereocilium is specialized may be consistent with current ideas that the transduction site at the apical pole of hair cells is located at the tips of the stereocilia (Hudspeth, 1982; 1986). Previously, it was proposed that transduction channels may be located on the apical membrane either at the base of stereocilia (Flock et al., 1977) or on the part of the membrane beneath which the cuticular plate is absent (Engstrom et al., 1962). In the present work, no local differentiation was apparent anywhere on the apical membrane, either in normal preparations or after exposure of tissue to filipin. It is thought that there may be only a relatively small number $(\sim 300)$ transduction channels on any one hair cell (Hudspeth, 1986). Consequently, if the transduction site(s) were present on the apical membrane it might be difficult to resolve but, as several other lines of evidence indicate that the tip region could be the site of the transduction channels, it would seem reasonable to suggest that the differentiation of the stereocilial membrane at the apical tip is associated with the presence of transduction channels in this region of the cell. If this is so, the large particles observed to be present in this region may represent the actual sites of the channels. The number of these on each stereocilium varied between two and up to eight on different stereocilia; it has

Fig. 14. IHC stereocilia following tomatin treatment. Most membranes have been cross-fractured, but where the membrane is exposed in face view, as at the arrow, membrane structure is seen to be distorted. Fractured; $\times 34400$.

Fig. 15. OHC stereocilia following tomatin treatment show very little effect, although the tomatin has clearly affected the apical membrane of cells (arrow). Fractured; $\times 25800$.

Fig. 16. P-fracture faces of apical-most tip region of $\mathrm{OHC}$ stereocilia in filipin-treated preparation. The tip region shows no filipin-induced lesions but a few large particles are apparent (arrows). Fractured; $\times 86000$.

Fig. 17. E-fracture face of the lower stereocilial tip of Fig. 16 in the complementary replica. The absence of filipin lesions is clearly apparent. The arrows indicate impressions on the e-face of three of the large tip particles exposed on the p-face. $x$ 113950.

Fig. 18. P-face of the apical-most tip of OHC stereocilia of the second stereociliary row following rotary replication of the specimen. Arrows indicate large particles clearly resolved at the tip. The absence of normal IMP in this region is also apparent. Fractured; $\times 193500$.

Fig. 19. Stereocilium of $\mathrm{OHC}$ in rapidly frozen preparation of the intact organ of Corti. The membrane at the apical end of the stereocilium is particle-free and the membrane contour is regular. Cleaved; $\times 86000$.

Fig. 20. Extracellular surface of the shaft of a stereocilium in a rapidly frozen preparation of isolated hair cells. Coarse granulations are irregularly distributed over the surface. Fractured; $\times 86000$.

Fig. 21. Extracellular surface at the tip of a stereocilium in a rapidly frozen isolated OHC. Fractured; $\times 86000$.

Fig. 22. Stereocilium from rapidly frozen preparation of isolated OHC. The fracture face shows particles similar to those in whole organ or Corti samples and the contour of the membrane is again regular. Fractured; $\times 51600$. 
been estimated that in the bull frog sacculus, the mean number of transduction channels on each stereocilium is approximately four (Hudspeth, 1986). However, this interpretation of the possible functional significance of the large tip particles must be purely conjectural until much more is known about the properties of the channels and the structure at the tip of the stereocilia has been examined in much greater detail.

\section{References}

BROWNELL, W. E. (1984) Microscopic observation of cochlear hair cell motility. Scanning Electron Microscopy 3, 1401-6.

CHANDLER, D. E. (1984) Comparison of quick-frozen and chemically fixed sea-ürchin eggs: Structural evidence that cortical granule exocytosis is preceded by a local increase in membrane mobility. Journal of Cell Science 72, 23-36.

DeROSIER, D. J., TILNEY, L. C. \& EGELMAN, E. (1980) Actin in the inner ear: the remarkable structure of the stereocilium. Nature 287, 291-6.

ENGSTROM, H., ADES, H. W. \& HAWKINS, J. E. (1962) Structure and functions of the sensory hairs of the inner ear. Journal of the Acoustical Society of America 34, 1356-63.

FLOCK, A. \& CHEUNG, H. C. (1977) Actin in sensory hairs of inner ear receptor cells. Journal of Cell Biology 75, $339-43$.

FLOCK, A., FLOCK, B. \& MURRAY, E. (1977) Studies on the sensory hairs of receptor cells in the inner ear. Acta Otolaryngologica 83, 85-91.

FORGE, A. (1985) Cholesterol distribution in cells of the stria vascularis of the mammalian cochlea and some effects of ototoxic diuretics. Journal of Cell Science 79, 181-97.

FORGE, A. (1986) The morphology of the normal cell membrane and junctional complexes of the cochlea. In: Basic and Applied Aspects of Noise-Induced Hearing Loss (edited by SALVI, R. J., HENDERSON, D., HAMERNIK, R. P. \& COLLETTI, v.) pp. 55-68. New York: Plenum Publishing Corporation.

FURNESS, D. N. \& HACKNEY, C. M. (1985) Cross-links between stereocilia in the guinea pig cochlea. Hearing Research 18, 177-88.

GULLEY, R. L. \& REESE, T. S. (1977) Regional specialization of the hair cell plasmalemma in the organ of Corti. Anatomical Record 189, 109-24.

HASTY, D. A. \& HAY, E. D. (1978) Freeze-fracture studies of the developing cell surface. II. Particle-free membrane blisters on glutaraldehyde fixed corneal fibroblasts are artefacts. Journal of Cell Biology 78, 756-68.

HIROKAWA, N. \& TILNEY, L. G. (1982) Interactions between actin filaments and between actin filaments and membranes in quick-frozen and deeply etched hair cells of the chick ear. Journal of Cell Biology 95, 249-61.

HUDSPETH, A. J. (1982) Extracellular current flow and the site of transduction by the vertebrate hair cells. Journal of Neuroscience 2, 1-10.

HUDSPETH, A. J. (1983) Mechanoelectrical transduction by hair cells in the acousticolateralis sensory system. Annual Review of Neuroscience 6, 187-215.

\section{Acknowledgements}

We thank Dr Lynn Carlisle for carrying out the quantitative analyses and for comments on the manuscript. We are grateful also to the BurroughsWellcome Trust for a travel grant to GZ and to both the Smithkline (1982) Foundation and the Hearing and Speech Trust (HAST) for equipment grants.

HUDSPETH, A. J. (1986) The ionic channels of a vertebrate hair cell. Hearing Research 22, 21-7.

KEMP, D. T. (1982) Cochlear echoes: Implications for noise-induced hearing loss. In: New Perspectives on Noise-Induced Hearing Loss (edited by HAMERNIK, R. P., HENDERSON, D. \& SALVI, R. J.). pp, 189-207. New York: Raven Press.

LIM, D. J. (1986) Functional structure of the organ of Corti: A review. Hearing Research 22, 117-46.

PHILLIPS, T. E. \& BOYNE, A. F. (1984) Liquid nitrogenbased quick freezing: experiences with a bounce-free delivery of cholinergic nerve terminals to a metal surface. Journal of Electron Microscopy Technique 1, 9-29.

PICKLES, J. O., COMIS, S. D. \& OSBORNE, M. P. (1984) Cross-links between stereocilia in the guinea pig organ of Corti, and their possible relation to sensory transduction. Hearing Research 15, 103-12.

PICKLES, J.O., COMIS, S. D. \& OSBORNE, M. P. (1986) The morphology of stereocilia and their cross-links in relation to noise damage in the guinea pig. In: Basic and Applied aspects of Noise-Induced Hearing Loss (edited by SALVI, R. J., HENDERSON, D., HAMERNIK, R. P. \& COLletti, V.), pp. 31-41. New York: Plenum Press.

RUSSELL, I. J. (1983) Origin of the receptor potential in inner hair cells of the mammalian cochlea - evidence for Davis' theory. Nature 301, 334-6.

RUSSELL, I. J., CODY, A. R. \& RICHARDSON, G. P. (1986) The responses of inner and outer hair cells in the basal turn of the guinea pig cohlea and in the mouse cochlea grown in vitro. Hearing Research 22, 199-216.

SANTI, P. A. \& ANDERSON, C. B. (1986) Alcian blue staining of cochlear hair cell stereocilia and other cochlear tissues. Hearing Research 23, 153-60.

SEVERS, N. J. \& ROBENEK, H. (1983) Detection of microdomains in biomembranes. An appraisal of recent developments in freeze-fracture cytochemistry. Biochimica et Biophysica Acta 737, 373-408.

SPOENDLIN, H. (1974) Neuroanatomy of the cochlea. In: Facts and Models in Hearing (edited by ZWICKER, E. \& TERHARDT, E.), pp. 18-32. Berlin: Springer-Verlag.

TILNEY, L. G. \& TILNEY, M. S. (1984) Observations on how actin becomes organized in cells. Journal of Cell Biology 99, 76s-82s.

ZAJIC, G. \& SCHACHT, J. (1987) Comparison of isolated outer hair cells from five mamalian species. Hearing Research 26, 249-56.

ZENNER, H. P., ZIMMERMANN, U. \& SCHMITT, U. (1985) Reversible contraction of isolated mammalian cochlear hair cells. Hearing Research 18, 127-33. 